\title{
Research on Design and Application of the IEC 61850-based Distribution Automation System
}

\author{
Hongwei Li ${ }^{1, a}$, Zhiyuan Pan ${ }^{1, b}$, Yubao Ren ${ }^{1, c}$, Ganrong Peng ${ }^{2 . d}$, Kui Tao ${ }^{3, e}$ \\ ${ }^{1}$ State Grid of China Technology College, Jinan, 250002, China \\ ${ }^{2}$ Jinan power supply company, Jinan, 250002, China \\ ${ }^{3}$ Lhasa power supply company, Lhasa, 850000 , China \\ adragonlhw@163.com, bz.y.pan@qq.com, ‘61142933@qq.com, dshmily1009@163.com, \\ etao4128271@qq.com
}

\begin{abstract}
Keywords: IEC 61850; distribution automation; plug and play; automatic transmission debugging; Information model mapping.

Abstract: With distribution automation full coverage, terminal debugging and operation and maintenance has become a key factor in practical application. Based on the IEC 61850 standard and IEC 61850-80-1, Information model mapping, combined with the IEC60870-5-104 extension, the scheme of self-discovery, self-registration and automatic transmission between distribution automation terminal and distribution automation master station system is put forward to realize the distribution terminal plug and play. The scheme is designed and developed in the master station system of distribution automation and distribution terminal. The feasibility and effectiveness of the scheme are proved by experiment.
\end{abstract}

\section{Introduction}

In accordance with the requirements of the Action Plan for Distribution Grid Construction \& Renovation 2015-2020 issued by the National Energy Administration, State Grid Corporation plans to carry out distribution automation construction in an all-round manner so that the distribution automation master system will cover all prefecture-level cities by 2018 and the total coverage of distribution automation of State Grid Corporation will reach more than $90 \%$ by the end of the $13^{\text {th }}$ Five-Year Planning Period; to be specific, the coverage of distribution automation of the provincial (district, city) branches east China will be no less than $95 \%$ and that of the provincial (district, city) branches in middle-west China will be no less than 90\%. China Southern Grid Corporation plans to bring the coverage of urban distribution grid automation to $80 \%$. Presently, the point table is checked manually for debugging between the distribution terminal device and the distribution automation master station system, which involves large workload $^{[1]}$ and growing number of distribution terminals connected, making it increasingly difficult to perform the debugging and operation \& maintenance work of the distribution automation system. With the advancements in distribution automation construction, the number of distribution terminals connected to the distribution automation master station has surged. As the distribution terminals come from different manufacturers, how to perform debugging between the distribution terminal and distribution automation master station system and active operation \& maintenance of the distribution terminal in a rapid, highly efficient and standardized manner to achieve plug-and-play of the distribution terminal devices has become one key factor affecting practical application of distribution automation. IEC 61850 provides a solution for plug-and-play of the distribution terminal through self-description of the distribution terminal by adopting the object-oriented data modeling. With the gradual maturity and extensive application of IEC 61850, its technologies and methods have been gradually applied in areas beyond substation automation $^{[2-3]}$, laying a foundation for application in the area of the distribution grid. IEC TC57 has initiated the plan to expand the application of IEC 61850 to the distribution grid and developed a raft of related standards to provide the standards and specifications ${ }^{[4]}$ for research and construction of the intelligent distribution grid. Also, there has been research on the application of IEC 61850 in 
distribution grid automation. Literature [5] applies IEC 61850 to the introduction of distribution grid automation, establishes the FTU information model and analyzes IEC 61850 service mapping to achieve communication between the distribution grid master station and the distribution terminal, and between the distribution terminal and the distribution terminal. Literature [6] comes up with the IEC 61850 standard based distribution terminal model on the basis of typical design of distribution automation equipment and technical specification for the distribution automation terminals. Literature[7] puts forward the Web Services + IEC 60870-5-104 mapping method by analyzing the application of the existing logic nodes of IEC61850 in distribution grid automation, establishing the FTU equipment model and analyzing the existing mapping methods of IEC 61850. Literature [8] comes up with the distribution terminal plug-and-play system based on IEC61850 and analyzes the model mapping method between the distribution automation master station system and the distribution terminal.

To standardize the transmission debugging process of the distribution terminal connected to the distribution automation master station system, reduce the debugging and operation \& maintenance workload and shorten the debugging and operation \& maintenance time and improve the operation $\&$ maintenance quality, this paper comes up with the scheme for self-discovery, self-registration and automatic transmission between the distribution terminal and the distribution automation master station system based on the IEC 61850 standard and the IEC 61850-80-1 information model mapping utilizing IEC 60870-5-101/104 extension to achieve plug-and-play of the distribution terminal.

\section{Overall Design Scheme}

Through analysis of the IEC 61850 international standard, the paper comes up with the scheme for plug-and-play of the distribution terminals. That is to say, the distribution terminal device has the self-description function based on IEC 61850 modeling, relying on service mapping of IEC 61850 and IEC 60870-5-101/104 and adopting file transfer to extend IEC 60870-5-101/104 communication protocols as communication support, and the distribution automation master station system is thus implemented to realize automatic discovery and automatic recognition of distribution terminals; Automatic association and mapping between the primary model and the secondary model is realized relying on automatic parsing to self-description information of distribution terminals by the distribution automation master station. Function of automatic transmission is designed on the basis of plug and play of distribution automation master station and distribution terminal so as to achieve the goal of standardized transmission debugging and in the meanwhile reduce the workload of transmission, operation and maintenance. The scheme includes three layers structurally, namely the master station layer of distribution automation, the access layer of distribution terminal and the distribution terminal layer. The master station layer of distribution automation mainly includes automatic mapping service of graphic model library, automatic transmission service, terminal registration and discovery service, data collection service, terminal management service and so on. The access layer of distribution terminal mainly includes distribution terminal registration and discovery services and data acquisition services. The distribution terminal layer mainly includes distribution modeling, registration and discovery service, communication service and so on. The overall structure is shown in Figure 1.

\section{Key Technology and Design}

Plug-and-play of the distribution terminal means that when the distribution terminal is newly installed or replaced on the site, the distribution automation master station can discover the distribution terminal actively, create the measurement model automatically based on the self-description information uploaded by the distribution terminal, and recognize the real-time telemetering and telesignaling information uploaded by the distribution terminal automatically. The key technologies of the plug-and-play of the distribution terminal mainly involve R \& D and design of service or function modules like self-registration and self-discovery of the distribution terminal, IEC 
61850 service mapping, measurement model mapping for graphical models and automatic transmission. To achieve plug and play of the distribution terminal, cooperation among various services is required. The plug and play flow charts between the distribution terminal and the master station of distribution automation are shown in Figure 2 and Figure 3.

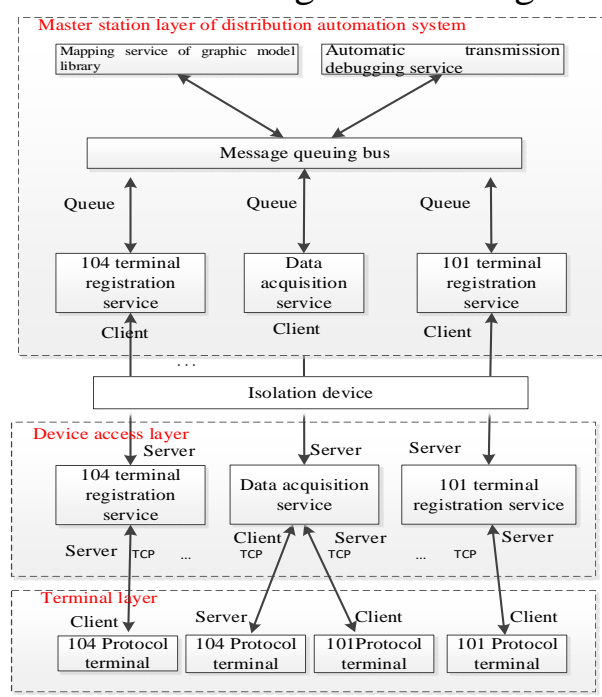

Fig.1 Structure of general scheme

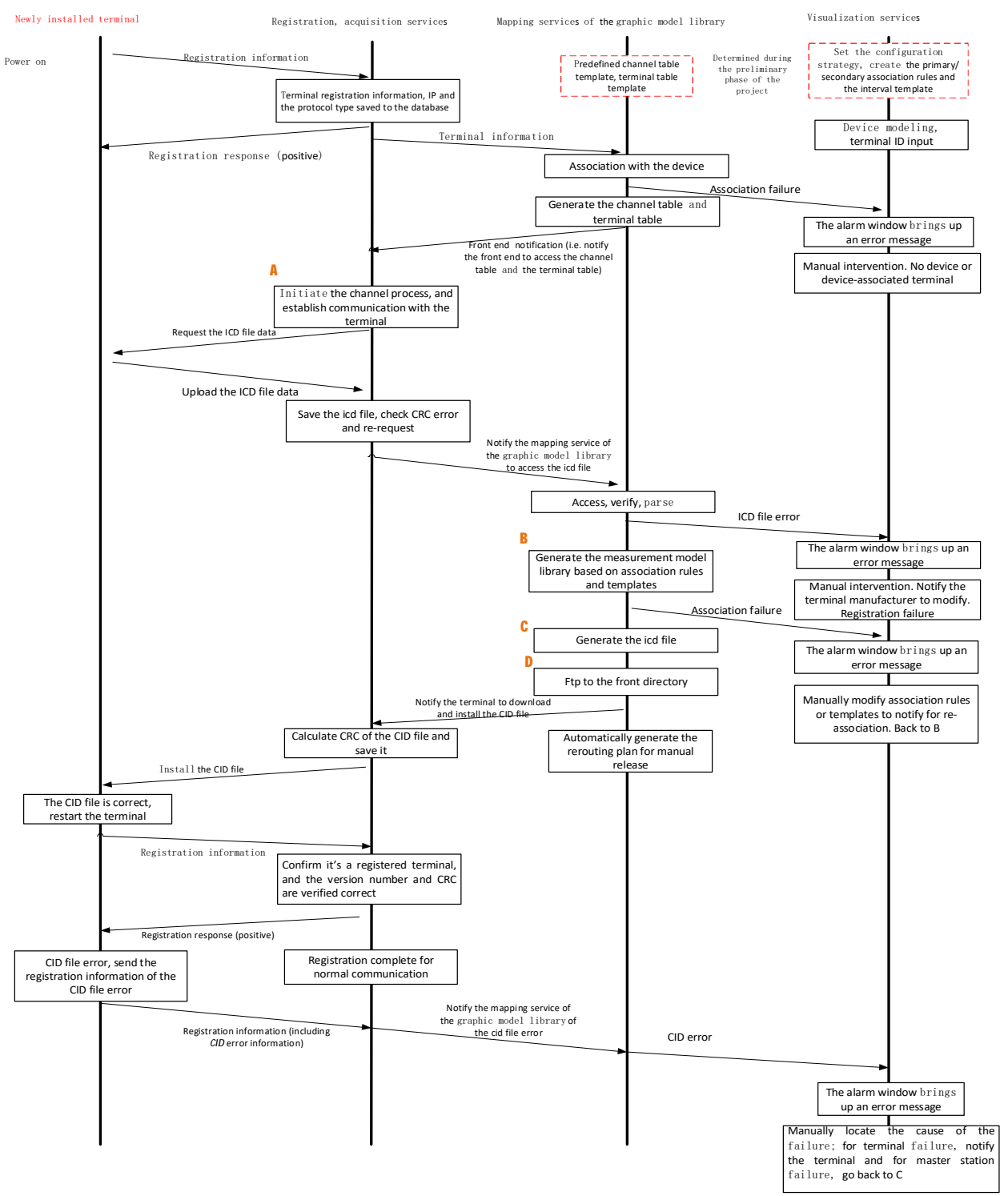

Fig.2 Plug and play flow chart about adding terminal 


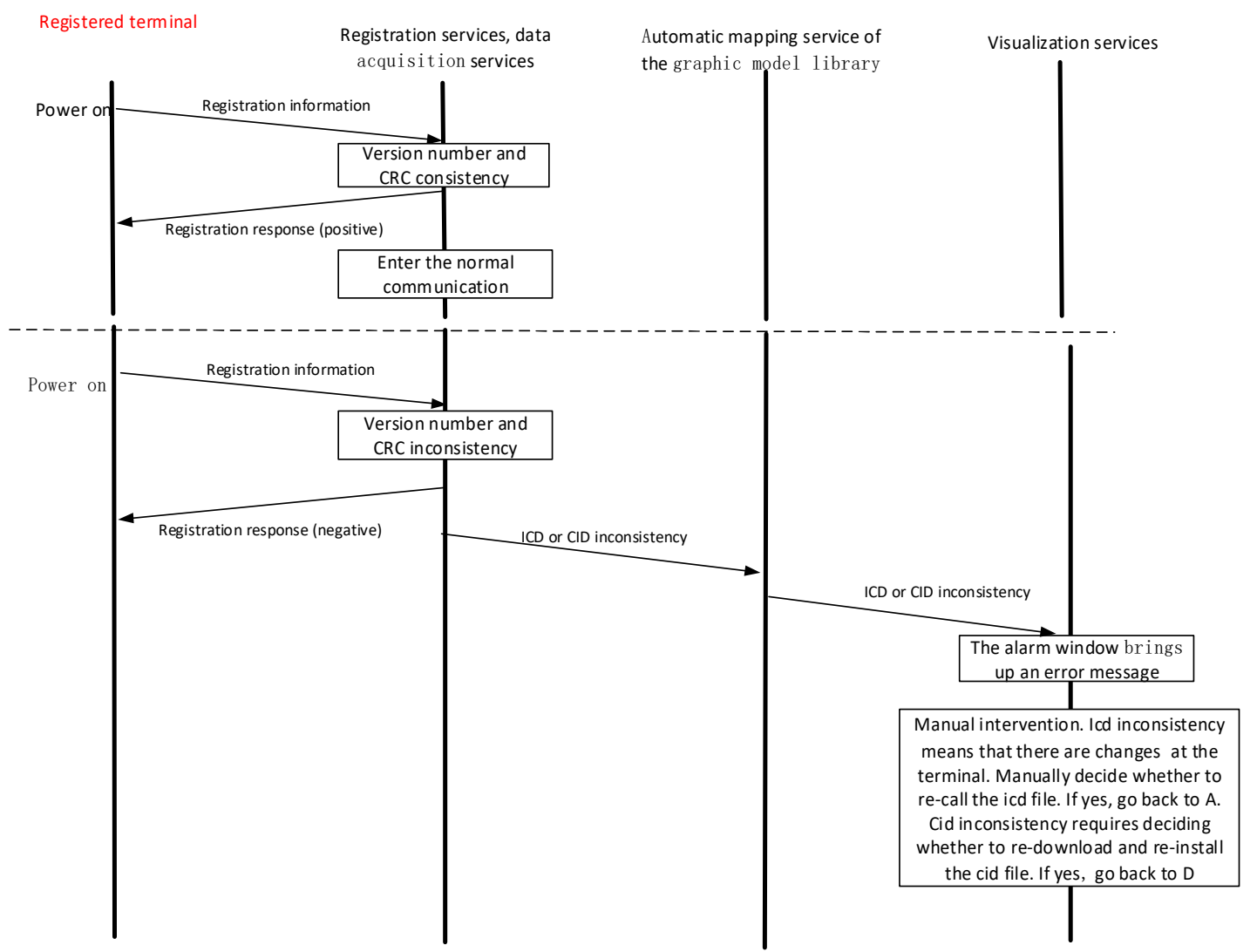

Fig.3 Plug and play flow chart about upgrading

Self-registration Service and Process. Once the distribution terminal is installed on the site and connected to the communication network, the distribution terminal establishes the connection to the master station of distribution automation and requests registration. After the master station of distribution automation receives the registration information and confirms its success, normal communication connection is established as shown in Fig.4.

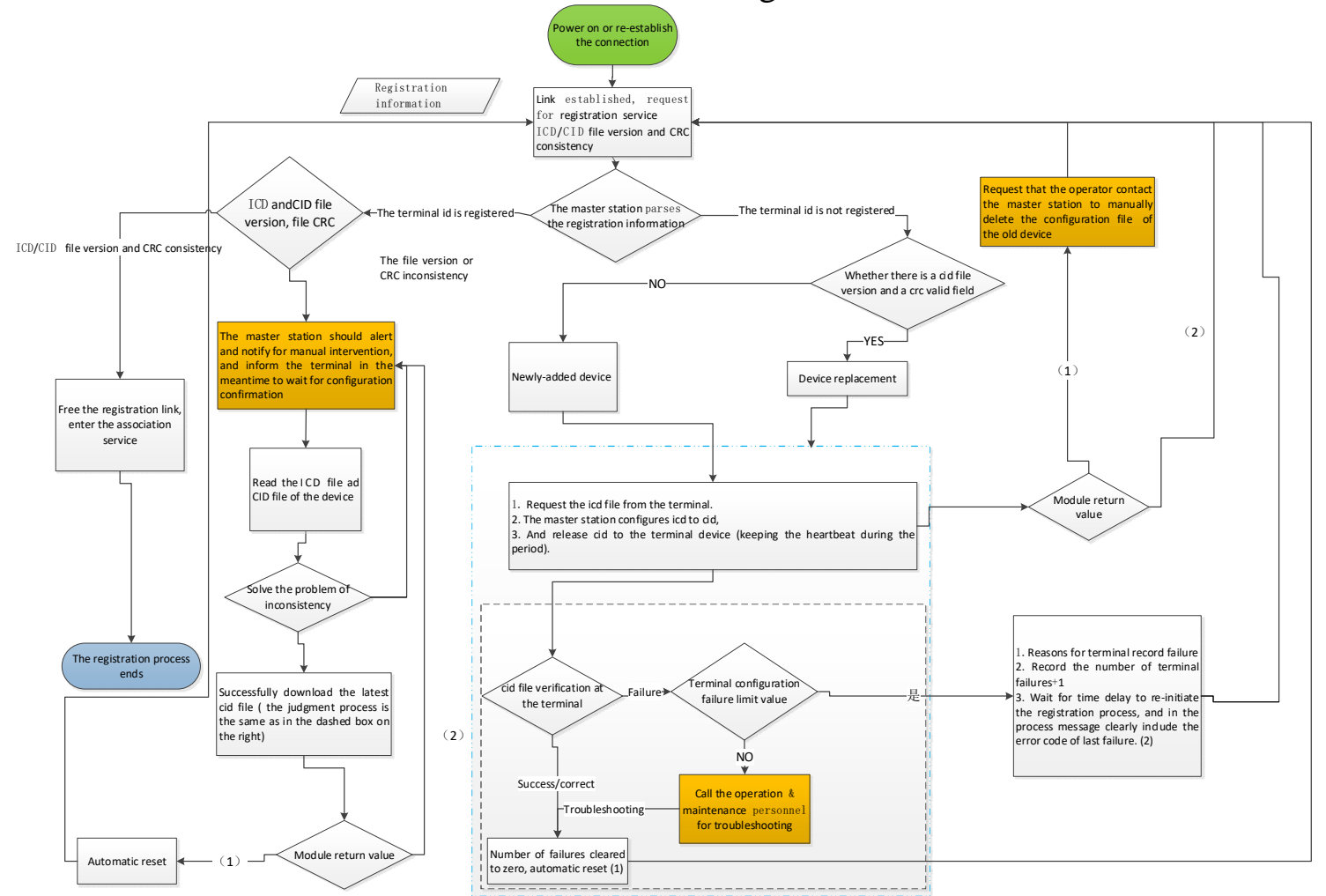

Fig.4 Registration flow chart 
Description of the registration mechanism and process:

$\boldsymbol{A}$. The master station receives the request information of the distribution terminal to determine whether it is a newly-added distribution terminal or not.

If it is determined as a newly-added terminal device, the master station of distribution automation will request the ICD file from the power distribution terminal actively. The master station generates CID from ICD and issues it to the distribution terminal. The distribution terminal checks the CID file. In case of success, the distribution terminal is automatically reset; in case of failure, the registration process will be re-initiated.

If it is determined as a registered distribution terminal and the file version or CRC is inconsistent, the master station of distribution automation sends out the alarm message and in the meanwhile notifies the distribution terminal to wait for configuration confirmation.

If it is determined as registered and the file version and CRC are consistent, the terminal will enter the normal handling process of 60870-5-101/104.

$\boldsymbol{B}$. For device replacement, the master station of distribution automation issues an alarm message. After the master station of distribution automation deletes the configuration information of the old distribution terminal, the registration process is the same as that of newly-added distribution terminals.

Service Mapping. Once the distribution terminal is installed on the site and connected to the communication network, the distribution terminal establishes the connection to the master station of distribution automation and requests registration. After the master station of distribution automation receives the registration information and confirms its success, normal communication connection is established as shown in Fig.4.

Currently the popular methods of IEC 61850 service mapping include mapping to manufacturing message specifications (MMS), mapping to IEC 60870-5-101/104 and mapping to Web Services. The three service mapping methods are analyzed in view of the fact that the MMS protocol is complicated with a huge system and a relatively big communication data flow, and its network requirements are higher ${ }^{[9]}$.

Web Services uses the unified and open standards of Internet, occupying more CPU and memory resources ${ }^{[7]}$ and it requires the establishment of a sound network information security mechanism and there are also other issues; IEC 60870-5-101/104 has the characteristics of small code quantity, low CPU utilization and strong real-time performance. Mapping to IEC 60870-5-101/104 is selected for service mapping. Although IEC 61850-80-1 defines the methods and data types of the IEC 61850 information model mapped to IEC 60870-5-101/104 in detail, the support for the service model is not sufficient. In this scheme, information is exchanged by adopting file transfer and expanding the user-defined marker for IEC 60870-5-101/104 as the service mapping and communication mechanism between the distribution terminal and distribution automation master station. File transfer is divided into two parts: file upload and file download (the processes are shown in Fig.5-1 and Fig.5-2). IEC 60870-5-101/104 shall be expanded according to application needs, for instance, acquiring the ICD file of the distribution terminal and installing the CID, SVG/CIM files, etc. into the distribution terminal by means of expanding IEC 60870-5-101/104 ASDU210.

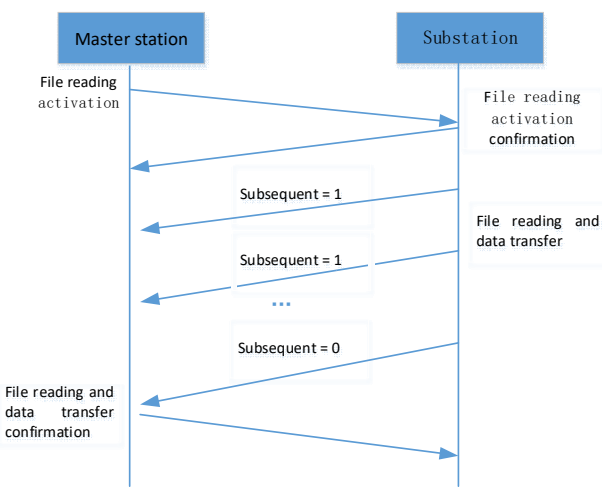

Fig.5-1 File upload flow chart

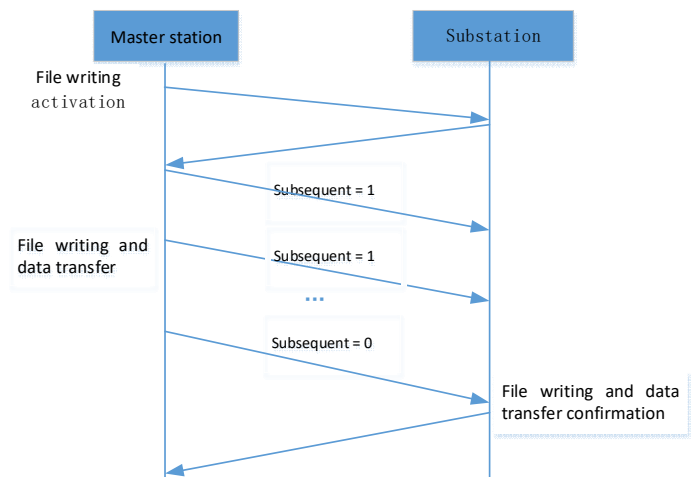

Fig.5-2 File download flow chart 
Graphic Model Library Automatic Mapping Service. The graphic model library automatic mapping function is a key factor in achieving automatic transmission and plug and play between the distribution automation master station and the distribution terminal. By parsing the self-description information uploaded by the distribution terminal and by employing the IEC 61850 and IEC61970 model mapping, the graphic model library automatic mapping service achieves association between primary and secondary devices and automatic creation of measurement model. As shown in Fig. 6, it mainly includes measurement template management, ICD file parsing management, CID file generation and download management, primary and secondary device association management, measurement library automatic generation management, exception alarm service and function module. Among them:

$\boldsymbol{A}$. Measurement template management: FTU and DTU measurement template customization and management, which are performed at station intervals.

B. ICD file parsing management: verify and parse the ICD files obtained from the distribution terminal, and save the parsing result to the graphic model library to provide data support for the measurement library in automatic mapping.

C. CID file generation and download management: generate CID file and download it to the distribution terminal through file transfer.

D. Primary and secondary device association management: achieve automatic matching and association between primary and secondary devices based on device UID in the self-description files of the distribution terminal and the ID of PMS imported into the distribution terminal. In addition, manual operation of device association is available.

$\boldsymbol{E}$. Measurement library automatic generation management: interact with the ICD file parsing management module to obtain distribution terminal device and measurement information, and interact with the measurement template management module to obtain the distribution device information, and perform automatic matching to allow for automatic generation of measurement library.

F. Exception alarm: give alerts about exceptions related to data parsing, verification and operation.

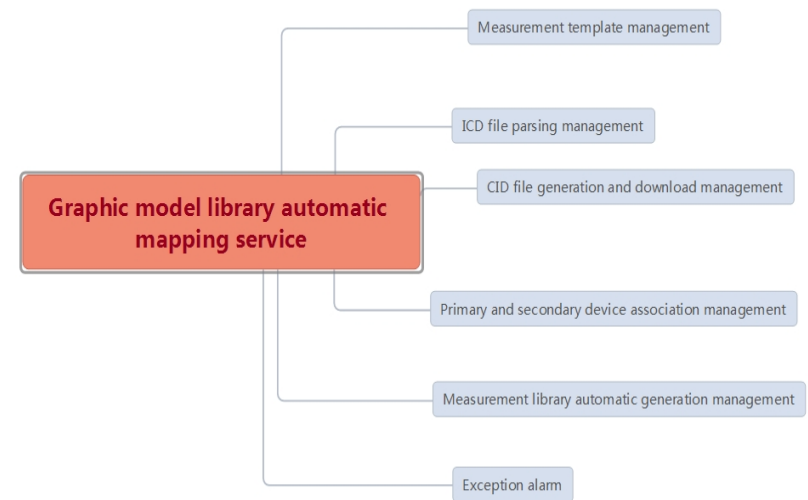

Fig.6 Structure of automatic map services about Measurement model

Automatic Transmission Service. In order to standardize the debugging and operation \& maintenance between the master station of distribution automation and the distribution terminal and reduce the debugging workload, the distribution terminal achieves automatic transmission by recognizing the real-time telemetering and telesignaling data uploaded by the distribution terminal automatically after automatic mapping with distribution self-registration, self-service and measurement model is completed. The automatic transmission service mainly includes such functions as visual display of the registration process, fixed parameter setting management and debugging report, etc. Among them:

A. Registration process visualization: to display the registration status, terminal related information, communication status and registration process information of the registered distribution terminal in a visual manner.. 
B. Fixed parameter setting and management: to maintain the template data, fixed data and terminal parameter, remotely set the terminal parameters and manage the setting process and historical data.

$\boldsymbol{C}$. Joint debugging report: automatically generate the distribution terminal joint debugging report, which covers the registration process report, communication establishing process report, point table check report, terminal information report and joint debugging conclusion report. The report information may be sent to the on-site O\&M debugging personnel via the SMS platform.

\section{Verification by Testing}

To verify the feasibility of the design, the test environment is built. The main hardware equipment of the test equipment includes 2 PC servers, 1 workstation, 1 switch, 3 distribution terminals and 1 short message module. Among them, the 2 PC servers are used for installing the TOSCAN-D3300C master station system of distribution automation, 1 workstation is used for visual display and the distribution terminal includes 1 FTU of FDR-115 feeder terminal, 1 DTU of the WPZD-163(III) station terminal and 1 DTU of the WPZD-135 intelligent low voltage district terminal. The structure of the test environment is shown in Fig. 7.

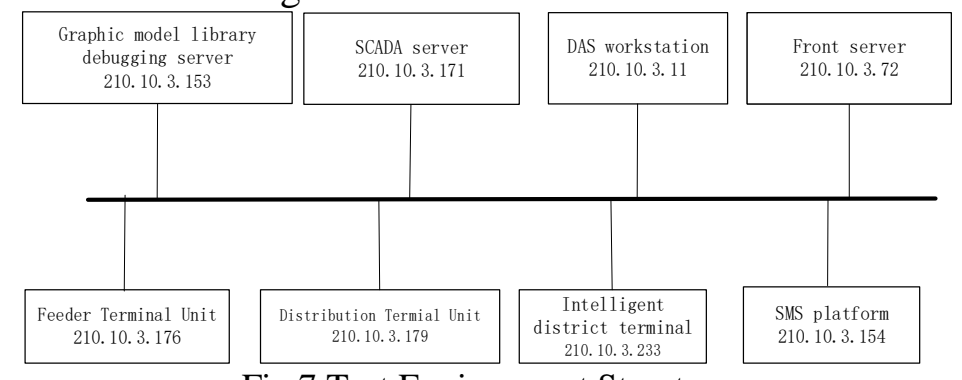

Fig.7 Test Environment Structure

The plug-and-play function of the 3 terminals: the FTU terminal, DTU terminal and low voltage intelligent terminal is tested in the test environment. The main test items include such functions as new terminal registration, terminal upgrade, automatic mapping of the measurement model, parameter setting table management and automatic transmission. Not only the functional correctness but also the feasibility of the plug-and-play function is verified. The terminal as the client sends the request for TCP connection to the server of the master station, which provides the registration service and listens to Port 12404.After the distribution terminal is connected to the network, the master station of distribution automation establishes a normal connection by acquiring the IP address and registration information of the distribution terminal. The master station system of distribution automation interprets and automatically maps based on the ICD file of the distribution terminal received to generate the secondary measurement model of the distribution equipment and completes the debugging of the distribution terminal equipment via the automatic transmission function.

\begin{tabular}{|c|c|c|c|}
\hline \multicolumn{4}{|c|}{ Terminal Managerment (F20XXXXXXX120201605081014)(01-01-02) } \\
\hline Terminal TC/TM/SV Point Table & \begin{tabular}{l|l|l} 
& Terminal parameter setting table & Regi
\end{tabular} & egistration process record & Parameter setting management \\
\hline No. & Process node & Status & Time \\
\hline 1 & Receive registration information & Success & 2016-11-08 16:36:47:002 \\
\hline 2 & Association with the primary device & Success & 2016-11-08 16:36:47:080 \\
\hline 3 & Generate communication configuration & Success & 2016-11-08 16:36:48:266 \\
\hline 4 & Acquire icd file & Success & 2016-11-08 16:37:19:833 \\
\hline 5 & Parse icd file & Success & $2016-11-08$ 16:37:20:442 \\
\hline 6 & Generate the TC/TM/SV Point Table & Success & 2016-11-08 16:37:21:004 \\
\hline 7 & Generate the parameter table & Success & $2016-11-08$ 16:37:21:690 \\
\hline 8 & Generate cid file & Success & $2016-11-08$ 16:37:22:378 \\
\hline 9 & cid file download & Success & 2016-11-08 16:38:12:857 \\
\hline 10 & Receive registration information(upgrade) & Success & 2016-11-08 16:42:43:193 \\
\hline 11 & Acquire icd file(upgrade) & Success & 2016-11-08 16:43:16:429 \\
\hline 12 & Parse icd file(upgrade) & Success & 2016-11-08 16:43:16:787 \\
\hline 13 & enerate the $\mathrm{TC} / \mathrm{TM} / \mathrm{sV}$ Point Table(upgrade) & Success & 2016-11-08 16:43:17:131 \\
\hline 14 & Generate the parameter table(upgrade) & Success & 2016-11-08 16:43:18:004 \\
\hline 15 & Generate cid file(upgrade) & Success & 2016-11-08 16:43:18:707 \\
\hline 16 & cid file download(upgrade) & Success & $2016-11-08$ 16:44:32:389 \\
\hline
\end{tabular}

Fig.8 Test results display 


\section{Conclusions}

The scheme builds the self-description of the distribution terminal based on IEC 61850, exchanges information by adopting file transfer and expanding the user-defined marker of IEC 60870-5-101/104 based on IEC 61850-80-1 information model mapping, develops secondary measurement model automatic mapping and automatic transmission service automatic debugging and therefore reduces the debugging installation workload. This scheme provides a plug-and-play method for the distribution terminals. Although the feasibility of the scheme is verified by testing, the scheme needs to be further optimized based on the on-site application scenarios and business requirements through application on the site.

\section{References}

[1] Xu Bingyin, Li Tianyou, Xue Yongduan, Intelligent Grid and Distribution Automation [J]. Power System Automaion,2009,33(17):38-41

[2] Li Yongliang, Li Gang.Brief Introduction to IEC 61850(Version 2) and Outlook of Its Application in Intelligent Grids [J]. 2010 Vol. 34(4): 11-16.

[3] IEC 61850-7-410 Hydroelectric power plants : communication for monitoring and control[s]

[4] Ren Yanming. IEC 61850 and Distribution Automation System. Power Supply \& Consumption,2014(5):30-33

[5]Han Guozheng, Xu Bingyin,Zhang Haitai. Application of IEC 61850 in Advanced Distribution Automation. Power Automation Equipment.2011(5),vol.31(5): 99-102.

[6] Zeng Jun, Zhou Jie, Xia Yandong, Liu Runmiao.Research on Standardized System Architecture of the Distribution Automation Terminal.Electric Appliances \& Energy Efficiency Management Technology,2015(9):20-25

[7]Han Guozheng,Xu bingyin,Suonan Jiale,Lü Guangxian,Wu Jianmin. Communication Technology for Distribution Automation Based on IEC 61850. Power System Protection and Control,2013 Vol.41(2):62-66

[8] Wu Huichao, Wu Yi, Zhu Haibing, Tan Zhijun, Liu Mingxiang. Research on IEC 61850-based Distribution Automation Terminal Plug-and-Play System. Power Supply \& Consumption,2015(01):60-63.

[9] Fang Xuezhi,Yang Fan,Xiao Bin,Yang Wanjin. Research and Development of Protective Device in Neutral Grounding via Low Resistance Based on IEC 61850 and Web services. Power System Protection and Control,2016,Vol 44,No 19:144-149 